\title{
EIGENSENSING AND DECONVOLUTION FOR THE RECONSTRUCTION OF HEAT ABSORPTION PROFILES FROM PHOTOACOUSTIC TOMOGRAPHY DATA
}

\author{
Zafer Doğan ${ }^{1,2}$, Thierry Blu ${ }^{3}$, and Dimitri Van De Ville , $^{1,2}$ \\ ${ }^{1}$ Medical Image Processing Lab (MIPLAB), Institute of Bioengineering, EPFL, CH-1015 Lausanne, Switzerland \\ ${ }^{2}$ Department of Radiology and Medical Informatics, University of Geneva, CH-1211 Geneva, Switzerland \\ ${ }^{3}$ Department of Electronic Engineering, Chinese University of Hong Kong, Shatin N.T. Hong Kong
}

\begin{abstract}
Photoacoustic tomography (PAT) is a relatively recent imaging modality that is promising for breast cancer detection and breast screening. It combines the high intrinsic contrast of optical radiation with acoustic imaging at submillimeter spatial resolution through the photoacoustic effect of absorption and thermal expansion. However, image reconstruction from boundary measurements of the propagating wave field is still a challenging inverse problem.

Here we propose a new theoretical framework, for which we coin the term eigensensing, to recover the heat absorption profile of the tissue. One of the main features of our method is that there is no explicit forward model that needs to be used within a (usually) slow iterative scheme. Instead, the eigensensing principle allow us to computationally obtain several intermediate images that are blurred by known convolution kernels which are chosen as the eigenfunctions of the spatial Laplace operator. The source image can then be reconstructed by a joint deconvolution algorithm that uses the intermediate images as input. Moreover, total variation regularization is added to make the inverse problem well-posed and to favor piecewise-smooth images.
\end{abstract}

Index Terms - Photoacoustic Tomography, Wave equation, source imaging, joint sparsity, deconvolution, total variation

\section{INTRODUCTION}

All imaging modalities have their strengths and drawbacks. To overcome the individual deficiencies and to combine their strengths, recently, a variety of the so called "hybrid methods" have been introduced and studied. Probably the most developed both experimentally and mathematically is photoacoustic tomography (PAT) [1]. Basically, photons are absorbed and create a thermally induced pressure jump that leads to ultrasonic waves that can be reconstructed with surprisingly high spatial resolution due to low scattering. PAT is non-ionizing, just as ultrasound CT, but its excitation process has been replaced by optical radiation that leads to clean and specific sources of pressure wave fields [2].

The major challenge in PAT resides in the reconstruction of the heat source function. From a physical point-of-view, PAT represents an inverse source problem belonging to the field of diffraction tomography. Therefore, various algorithms used in PAT have borrowed from other imaging modalities such as ultrasound, x-ray and optical tomographies. Numerous approaches have been suggested to reconstruct the initial pressure rise to the photoacouctic effect in the tissue due to absorbed incident light. Among others, Radon

This work was supported in part by the Swiss National Science Foundation (under the grants 2053530-132808 and PP00P2-123438) and in part by the Center for Biomedical Imaging (CIBM) of Geneva-Lausanne Universities and Hospitals, and the EPFL. transform approaches [3], back-projection algorithm [4], Fourier and time-domain algorithms are all successful in recovering the source function, but mostly they require extensive computation of the forward problem within an iterative framework.

From a mathematical point-of-view, PAT belongs the inverse problem of generalized spherical Radon transform. In general, this approach provides a satisfactory result only for the regions that are near the center of the detection geometry where the approximation quality of the Radon transform does not deviate too much from the approximation. Xu et al. [5] explained what parts of the image can be stably recovered in the limited-view imaging theoretically. Finch et al. [6] showed exact inverse solutions based on the Radon transform approximation with full-view data.

Numerous approaches have been proposed for the inverse problem of PAT in discrete form. The standard linear system solutions are incorporated in the reconstruction of the source function [7] mostly through iterative algorithms. Compared with the approximation methods, iterative techniques give more accurate results, in particular, when prior knowledge about the source function is known and used to regularize the solution [8]. Finch et al. [9] recently reported a filtered back-projection type algorithm to recover the initial source function from spherical means of the wave field over spheres centered on the boundary of the measurement surface.

In most of the recent work, the sparsity has played a key role in regularizing the solution for the inverse problem. In particular, point source localization methods have also been developed and applied in this setting [10]. Ammari et al. [11] developed the mathematical modeling in PAT of small absorbers and propose MUSIC-type of algorithms for the estimation of the location of the absorbing regions. Badia and Nara [12] reported the uniqueness and the local stability of the point source localization problem.

In this paper, we propose a new reconstruction framework to extract the optical absorption profile of the breast tissue with high accuracy from PAT data. The proposed framework allows for efficient recovery of the local absorption image of the breast tissue without explicit forward model of the wave propagation. Moreover, any prior knowledge about the characteristics of the image can be incorporated into a regularization framework that allows the recovery of smooth and fine detailed images.

The paper is organized as follows. In Section 2, we introduce the inverse problem of PAT. In Section 3, we provide the fundamentals of Eigenensing framework. In Section 4, we develop the the proposed method for a 2D imaging setup. In Section 5, the feasibility of the porposed method is demonstrated with a $2 \mathrm{D}$ numeric phantom representing spatial absorption map of a breast. 


\section{INVERSE SOURCE PROBLEM IN PAT}

Optical radiation absorbed by the tissue deposits thermal energy. Due to the photoacoustic effect, the subsequent thermal expansion can be modeled as a heat source $H(\mathbf{r}, t)$ that gives rise a pressure field $u(\mathbf{r}, t)$ governed by the wave equation in an acoustically homogeneous medium: (under the condition of thermal confinement)

$$
\nabla^{2} u(\mathbf{r}, t)-\frac{1}{c^{2}} \frac{\partial^{2} u(\mathbf{r}, t)}{\partial t^{2}}=-\frac{\beta}{C_{p}} \frac{\partial}{\partial t} H(\mathbf{r}, t),
$$

where $\beta$ is the isobaric volume expansion coefficient $\left[\mathrm{K}^{-1}\right], C_{p}$ is the specific heat $[\mathrm{J} /(\mathrm{K} \mathrm{kg})]$, and $c$ is the speed of sound. Human breast is made of soft tissue with speed variations in the range of $10 \%$ and can thus be considered as an homogeneous medium [2]. The heating function can be further decomposed as the product

$$
H(\mathbf{r}, t)=A(\mathbf{r}) g_{e}(t),
$$

where $A(\mathbf{r})$ is the spatial absorption function and $g_{e}(t)$ the temporal illumination function, which is known by the optical excitation protocol. The spatial absorption function is to be recovered from the boundary measurements of the pressure wave $u$.

\section{FUNDAMENTALS OF EIGENSENSING}

We further assume that the region-of-interest is $\Omega$ with boundary $\partial \Omega$. We can then write the time harmonic solutions of the PAT wave equation as

$$
\nabla^{2} U(\mathbf{r}, \omega)+\frac{\omega^{2}}{c^{2}} U(\mathbf{r}, \omega)=-(j \omega) A(\mathbf{r}) G_{e}(\omega),
$$

which is the inhomogeneous Helmholtz equation. The problem at hand is to recover the source function $A(\mathbf{r})$ from the Cauchy data $\left(\left.U\right|_{\partial \Omega}, \nabla U \cdot \mathbf{e}_{\partial \Omega}\right)$, which consists of the pressure field and its normal derivative along $\mathbf{e}_{\partial \Omega}$ being the unit outward normal to the boundary, respectively.

Without loss of generality, we can now consider a specific frequency $\omega$ with corresponding wave-number $k=\omega / c$. We also denote $\alpha=(j \omega) G_{e}(\omega)$. From Green's second identity for the wave field $U$ and a test function $\Psi(\mathbf{r})$, we obtain a link between the source term and the surface measurements as follows

$$
\int_{\Omega}\left(U \nabla^{2} \Psi-\Psi \nabla^{2} U\right) d V=\oint_{\partial \Omega}(U \nabla \Psi-\Psi \nabla U) \cdot \mathbf{e}_{\partial \Omega} d S .
$$

Next we introduce the source distribution in the left hand-side by using (2), resulting in,

$\int_{\Omega}\left[U\left(\nabla^{2} \Psi+k^{2} \Psi\right)+\alpha A \Psi\right] d V=\oint_{\partial \Omega}(U \nabla \Psi-\Psi \nabla U) \cdot \mathbf{e}_{\partial \Omega} d S$.

By construction, we then choose the test function $\Psi$ such that it is a "free space" solution of the homogeneous Helmholtz equation

$$
\nabla^{2} \Psi+k^{2} \Psi=0,
$$

which is equivalent to $\Psi$ being an eigenfunction of the spatial Laplace operator $\nabla^{2} \Psi=\lambda \Psi$, with $\lambda=-k^{2}$. Therefore, we coin the term "eigensensor" for $\Psi$. Additionally, since the Laplace operator is shift-invariant, we can "sense" the source function at different spatial positions and construct the sensing function

$$
\begin{aligned}
I_{\Psi}(\mathbf{r}) & =\frac{1}{\alpha}(A * \alpha \Psi)(\mathbf{r}) \\
& =\int_{\Omega} \Psi\left(\mathbf{r}-\mathbf{r}^{\prime}\right) A\left(\mathbf{r}^{\prime}\right) d \mathbf{r}^{\prime} \\
& =\frac{1}{\alpha} \oint_{\partial \Omega}\left[U \nabla \Psi\left(\mathbf{r}-\mathbf{r}^{\prime}\right)-\Psi\left(\mathbf{r}-\mathbf{r}^{\prime}\right) \nabla U\right] \cdot \mathbf{e}_{\partial \Omega} d S,
\end{aligned}
$$

where $*$ is the 2-D or 3-D convolution operator and we have compensated for the factor $\alpha$ (which is known). We can thus compute the sensing image $I_{\Psi}$ at any $\mathbf{r}$ by the surface integral (7) using the boundary measurements, which represents an interaction (6) of the source function with the eigensensor. In sum, the boundary measurements have been mapped to volumetric information without an explicit forward model as in conventional methods.

\section{2D IMAGE RECONSTRUCTION}

To demonstrate the feasibility of the proposed method, we developed our method in an instructive 2-D setting. Let us first derive the separable eigenfunction of the 2-D Laplace operator in polar coordinates $(r, \phi)$ :

$$
\left[\frac{1}{r} \frac{\partial}{\partial r}\left(r \frac{\partial}{\partial r}\right)+\frac{1}{r^{2}} \frac{\partial^{2}}{\partial \phi^{2}}\right] \Psi(r, \phi)=-k^{2} \Psi(r, \phi) .
$$

Using the separation of variables method and the non-singularity requirement at the origin, we get the eigenfunction of the Laplace operator in polar coordinates as the basis function for the polar Fourier transform on the whole space

$$
\Psi_{m}^{k}(r, \phi)=\sqrt{\frac{k}{2 \pi}} J_{m}(k r) e^{j m \phi}
$$

where $J_{m}$ are the $\mathrm{m}^{\text {th }}$-order Bessel functions $(m=0,1, \ldots)$. To better appreciate the meaning of the eigensensors, we provide the Fourier transform of $\Psi_{m}$

$$
\mathcal{F}\left\{\Psi_{m}^{k}\right\}\left(k^{\prime}, \varphi_{k}^{\prime}\right)=\delta\left(k-k^{\prime}\right) \frac{(-i)^{m}}{\sqrt{2 \pi k}} e^{i m \varphi_{k}^{\prime}},
$$

where $k^{\prime}$ and $\varphi_{k}^{\prime}$ are the radial and angular parts of the polar Fourier space and which is nonzero only on a circle of radius $k$. Alternatively, this states that plane waves of the same wavenumber, as the basis function for the normal Fourier transform, can be superposed to get a so-called cylindrical wave.

We can then formulate the image reconstruction problem as a joint deconvolution of multiple sensing functions degraded by known eigensensors by means of their known frequency domain localization. In particular, given the set of sensing functions $I_{\Psi_{m}^{k_{n}}}$ computed from the measurements using eigensensors $\Psi_{m}^{k_{n}}$, $m \stackrel{m}{=} 1, \ldots, M$ and at finitely chosen photoacoustically measurable wavenumbers such that $0<k_{l} \leq k_{n} \leq k_{h}, n=1, \ldots, N$ where $k_{l}$ and $k_{h}$ represents the lower and upper limits of $k$, we solve for

$$
\hat{A}=\underset{A}{\arg \min } \sum_{n=1}^{N} \sum_{m=1}^{M}\left\|I_{\Psi_{m}^{k_{n}}}-A * \Psi_{m}^{k_{n}}\right\|_{2}^{2}+\mu J_{\mathrm{reg}}(A)
$$

where the first term measures the data fidelity (under the $\ell^{2}$-norm) and the second term regularizes the source function with parameter $\mu \geq 0$ controlling the relative weights of the two terms. The regularization term is used to favor some solutions by penalizing the other by measuring the lack of smoothness and sparseness with differential operators. We choose the regularization term as the total variation (TV) semi-norm

$$
J_{\mathrm{TV}}(A)=\int_{\mathbb{R}^{2}}\|\nabla A(\mathbf{r})\|_{2} d \mathbf{r}
$$

which favors sparseness in the gradient of $A$. Recent advances in convex optimization theory have led to efficient algorithms for such minimization problem. 


\subsection{Minimization Algorithm}

The main difficulty in solving (11) is due to the non-differentiability of the regularization term. Standard approaches are to approximate the TV semi-norm with a smooth and differentiable one or to approximate it with a quadratic penalty technique. Here, we choose to use the alternating direction method of multipliers (ADMM) for which convergence has been proved [13]. For the sake of completeness, we briefly review the application of the ADMM method for the current problem.

Hereafter, we assume a discrete setting and replace (11) with the following problem noticing that eigensensors for the same order $m$ are mutually disjoint in the frequency domain

$$
\hat{a}=\underset{a}{\arg \min } \frac{1}{2} \sum_{m=1}^{M}\left\|\mathcal{F} I_{\Gamma_{m}}-\Gamma_{m} \mathcal{F} a\right\|_{2}^{2}+\mu \sum_{i}\left\|D_{i} a\right\|_{2}
$$

where $a$ is an $n \times n$ gray-scale image representing the absorption map, $\mathcal{F}$ is a two-dimensional discrete Fourier transform matrix and $\Gamma_{m}=\sum_{n=1}^{N} \mathcal{F} \Psi_{m}^{k_{n}}$ is the Fourier transform of $\mathrm{m}^{\text {th }}$-order joint eigensensor, i.e., it represents a weighted selection matrix in $k$-space according to (10) and $I_{\Gamma_{m}}=\sum_{n=1}^{N} I_{\Psi_{m}^{k_{n}}}$ is the $\mathrm{m}^{\text {th }}$-order observation of $a$. Next, $\sum_{i}\left\|D_{i} a\right\|_{2}$ is a discretization of the total variation (TV) of $a$ with the common TV-L2 model where $D_{i} a=$ $\left[\left(D^{(1)} a\right)_{i} ;\left(D^{(2)} a\right)_{i}\right]$ denotes the discrete gradient of $a$ at pixel $i$ with $D^{(1)}$ and $D^{(2)}$ representing the two first order forward finite difference operators under periodic boundary conditions for $a$.

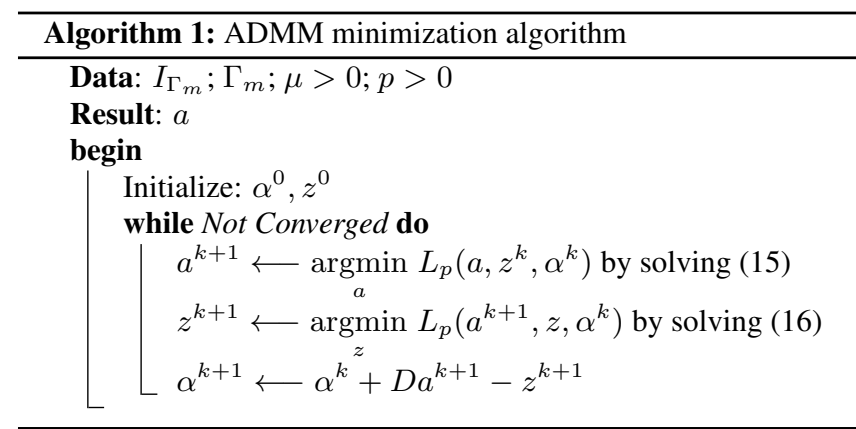

Next, we first introduce auxiliary variables $z_{i}=\left(\left(z_{1}\right)_{i} ;\left(z_{2}\right)_{i}\right)$ 's which is an approximation of $D_{i} a$ and then replace (13) with a constrained minimization problem

$\min _{a, z_{i}} \frac{1}{2} \sum_{m=1}^{M}\left\|\mathcal{F} I_{\Gamma_{m}}-\Gamma_{m} \mathcal{F} a\right\|_{2}^{2}+\mu \sum_{i}\left\|z_{i}\right\|_{2} \quad$ s.t. $z_{i}=D_{i} a, \forall i$.

Relaxing the equality conditions and penalizing their violations by quadratic functions we get the scaled form [13] of the corresponding augmented Lagrangian

$$
\begin{aligned}
L_{p}(a, z, \alpha)=\frac{1}{2} & \sum_{m=1}^{M}\left\|\mathcal{F} I_{\Gamma_{m}}-\Gamma_{m} \mathcal{F} a\right\|_{2}^{2}+\mu \sum_{i}\left\|z_{i}\right\|_{2} \\
& +\frac{p}{2}\|D a-z+\alpha\|_{2}^{2}
\end{aligned}
$$

where $\alpha$ is a scaled dual variable and $p$ is the penalty parameter. Despite more decision parameters compared to (13), problem (14) is easier to minimize with respect to $z$ and $a$. First for a fixed $z$, the minimization of (14) for $a$ becomes the least square problem

$$
\min _{a} \frac{1}{2} \sum_{m=1}^{M}\left\|\mathcal{F} I_{\Gamma_{m}}-\Gamma_{m} \mathcal{F} a\right\|_{2}^{2}+\frac{p}{2}\|D a-z+\alpha\|_{2}^{2} .
$$

Secondly, for a fixed $a$, the second and third terms are separable with respect to $z_{i}$, so minimizing (14) for $z$ is equivalent to solving

$$
\min _{z_{i}}\left(\left\|z_{i}\right\|_{2}+\frac{p}{2}\left\|D_{i} a-z_{i}+\alpha\right\|_{2}^{2}\right), \forall i,
$$

for which the minimizer is given by the two dimensional shrinkage formula[14]. We provide a summary of the method in Algorithm 1.

\section{EXPERIMENTAL RESULTS}

For PAT breast imaging system, the detection geometry is typically assumed to have a $8 \mathrm{~cm}$ radius and the generated pressure field is sampled with fast ultrasound transducers capable of linear and planar measurements of the wave field. The surface integral (5), which is the core of the eigensensing framework, can be approximated thanks to the high sampling densities that are achieved in current devices [2]. For signals in the typical PAT range of $0.1-3 \mathrm{MHz}$, the wavenumber $k$ will be between 500-12'500/m assuming constant speed of sound in soft tissue,i.e., $c=1500 \mathrm{~m} / \mathrm{s}$ which imposes approximately $0.4 \mathrm{~mm}$ spatial resolution of the sensing functions to be constructed.

We perform numerical experiments to evaluate the performance of our reconstruction algorithm on a numerical phantom of size $4096 \times 4096$ for the absorption map of a $16 \mathrm{~cm} \times 16 \mathrm{~cm}$ breast tissue given in Fig. 1 (a). We choose only the zeroth order eigenfunction $\Psi_{0}^{k}$ in (9) for different wavenumber, $k$ from the specified PAT range. In our framework, each eigenfunction will perform a selection of partial Fourier measurements only on a circle of radius $k$ in the k-space.

Due to incapability of most standard ultrasound transducers, detectors miss the low frequency components- frequencies less than $0.1 \mathrm{MHz}-$ that are necessary for characterization of different tissues. Therefore, we propose to compare the reconstruction result and the original absorption map after a normalization process. We coin the term SNR improvement (iSNR)

$$
\mathrm{iSNR}=10 \log _{10} \frac{\sum_{i}\|\overline{\hat{a}}-\bar{a}\|^{2}}{\sum_{i}\left\|\overline{\hat{a}}_{0}-\bar{a}\right\|^{2}},
$$

where $a, \hat{a}$ and $\hat{a}_{0}$ are the original image, the reconstructed image and the initial back-projected image, respectively, which are all normalized with respect to the mean and standard deviation of the data, i.e., $\bar{a}=(a-\eta) / \sigma$ where $\eta$ is the mean and $\sigma$ is the standard deviation of the data.

For the experiments, we use the breast phantom in Fig. 1 (a) and the image is scaled to $[0,1]$ followed by a degradation with additive white gaussian noise at $20 \mathrm{~dB}$. We assume that the surface integral (7), is well-approximated and we solve for (13) for a regularization parameter $\mu=8$ using partial Fourier samples along a number of circles that corresponds to chosen wavenumbers from the range of PAT. From Table 1, we demonstrate the results of the reconstruction quality with respect to number of evenly chosen frequencies on the Fourier domain. As it is expected, the quality of the reconstruction

Table 1: Comparison of reconstruction quality in terms of iSNR with respect to number of wavenumber used (\#k) evenly spaced on the $\mathrm{k}$-space in the range of PAT.

\begin{tabular}{c|c|c|c}
\hline \hline$\# \mathrm{k}$ & iSNR $(\mathrm{dB})$ & $\# \mathrm{k}$ & $\mathrm{iSNR}(\mathrm{dB})$ \\
\hline 5 & 3.19 & 30 & 6.26 \\
\hline 10 & 4.74 & 35 & 6.27 \\
\hline 15 & 5.79 & 40 & 6.28 \\
\hline 20 & 5.88 & 45 & 6.29 \\
\hline 25 & 6.21 & 50 & 6.30 \\
\hline \hline
\end{tabular}




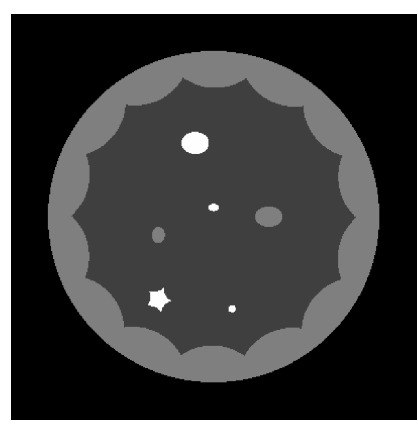

(a)

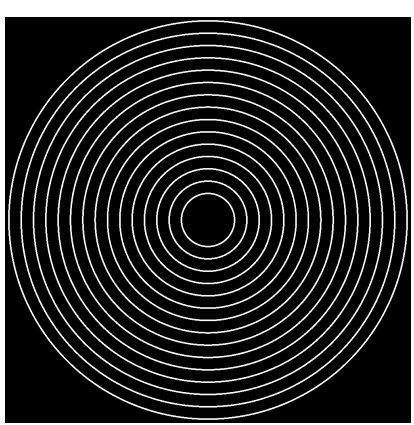

(b)

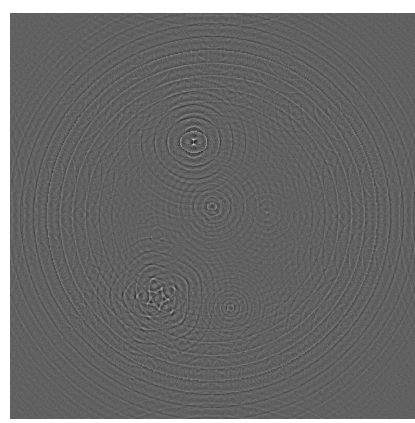

$(c)$

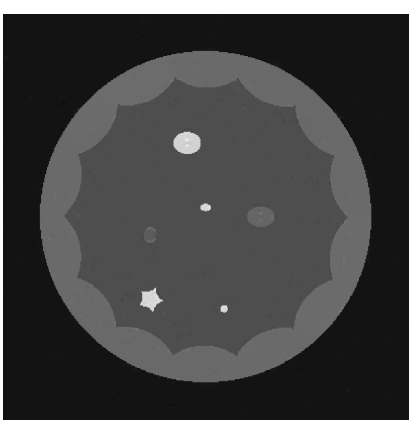

(d)

Fig. 1: Reconstruction of heat absorption profile of a breast phantom; (a) original phantom (b) partial frequency sampling along 15 evenly chosen wavenumbers from the range of PAT using eigensensing principle, (c) inverse Fourier transform of the measured partial Fourier data with iSNR 0dB (d) reconstruction with total variation $(\mathrm{TV})$ regularization iSNR $5.79 \mathrm{~dB}$

increases as the the number of chosen wave numbers increases which allows more coverage of partial Fourier measurements. The results in Table 1 also released that the necessary numbers of wavenumbers to be be used are limited for images composed of piecewise smooth absorption regions.

In Fig. 1, we show the reconstruction result for a case where we evenly select 15 frequencies from the k-space. We observe the corresponding joint mask in the Fourier plane in Fig. 1 (b) and the corresponding inverse Fourier of the measured partial frequency data with $0 \mathrm{~dB}$ iSNR by (17). Finally, in Fig. 1 (d), we provide our solution with $5.79 \mathrm{~dB}$ iSNR with respect to normalized data as explained earlier. The result shows that reconstruction of fine spatial details while preserving sharp boundaries is achieved with the proposed method thanks to the TV regularization which is well-suited to the purpose of reconstruction of images composed of piecewise smooth regions.

\section{CONCLUSIONS}

We presented a novel theoretical framework called eigensensing principle and its application to PAT to recover the heat absorption profile of the breast tissue. We proposed a family of sensing functions being an eigenfunction of the spatial Laplace operator to relate the measurements of photoacoustically generated wave field to the spatial absorption map of the tissue. We also showed that the eigensensing principle allows for partial Fourier domain measurements that can be used in a joint deconvolution framework. The choice of the sensing functions to be the eigenfunction of the spatial Laplace operator in polar coordinates allowed better Fourier plane coverage per sensing function rather than choosing a plane wave. Notice that Bessel functions in the proposed sensing function asymptotically decay like $1 / \sqrt{r}$ which yields better spatial localization than a plane wave which also improves the approximation of the surface integration of the measurements with the sensing function.

We applied our method on a numerical phantom representing the heat absorption profile of a breast tissue for a 2-D proof- of-concept study. We chose total variation (TV) semi-norm as the regularization term that favors sparseness in the gradient of the absorption profile as a priori. The preliminary results showed that eigensensing principle yields promising results on detection of fine spatial details with varying absorption characteristics which is of great importance in distinguishing malignant breast tissued from normal ones.

Sparse image models for the reconstruction of the absorption profile the breast tissue in PAT from overdetermined boundary field measurements remains as a promising research area of further research. Moreover, we also consider possibility and feasibility of the proposed method for real PAT data for imaging heat absorption profile of the breast tissue.

\section{REFERENCES}

[1] L. V. Wang and S. Hu, "Photoacoustic tomography: In vivo imaging from organelles to organs," Science, vol. 335, no. 6075, pp. 1458-1462, 2012.

[2] M. Xu and L. Wang, "Photoacoustic imaging in biomedicine," Rev. Sci. Instrum., vol. 77, no. 4, pp. 041101, 2006.

[3] K. Wang and M. Anastasio, "Photoacoustic and thermoacoustic tomography: Image formation principles," in Handbook of Mathematical Methods in Imaging, O. Scherzer, Ed., pp. 781-815. Springer New York, 2011.

[4] M. Xu and L. V. Wang, "Universal back-projection algorithm for photoacoustic computed tomography," Phys. Rev. E, vol. 75, pp. 059903, May 2007.

[5] Y. Xu, L. V. Wang, G. Ambartsoumian, and P. Kuchment, "Reconstructions in limited-view thermoacoustic tomography," Medical Physics, vol. 31, no. 4, pp. 724-733, 2004.

[6] D. Finch and S. Patch, "Determining a function from its mean values over a family of spheres," SIAM Journal on Mathematical Analysis, vol. 35, no. 5, pp. 1213-1240, 2004.

[7] G. Paltauf, J. A. Viator, S. A. Prahl, and S. L. Jacques, "Iterative reconstruction algorithm for optoacoustic imaging," The Journal of the Acoustical Society of America, vol. 112, no. 4, pp. 1536-1544, 2002.

[8] L. V. Wang and H.-I. Wu, "Biomedical optics, principles and imaging," Journal of Biomedical Optics, vol. 13, no. 4, pp. 049902-049902-2, 2008.

[9] M. Haltmeier, D. Finch, and Rakesh, "Inversion of spherical means and the wave equation in even dimensions," SIAM Journal of Applied Mathematics, vol. 68, no. 2, pp. 392-412, 2007.

[10] Z. Dogan, I. Jovanovic, T. Blu, and D. Van De Ville, “3d reconstruction of wave-propagated point sources from boundary measurements using joint sparsity and finite rate of innovation," in Biomedical Imaging (ISBI), 2012 9th IEEE International Symposium on, may 2012, pp. $1575-1578$.

[11] H. Ammari, E. Bossy, V. Jugnon, and H. Kang, "Mathematical modeling in photoacoustic imaging of small absorbers," SIAM Rev., vol. 52, pp. 677-695, November 2010.

[12] A. E. Badia and T. Nara, "An inverse source problem for Helmholtz's equation from the Cauchy data with a single wave number," Inverse Problems, vol. 27, no. 10, pp. 105001, 2011.

[13] S. Boyd, N. Parikh, E. Chu, B. Peleato, and J. Eckstein, "Distributed optimization and statistical learning via the alternating direction method of multipliers," Found. Trends Mach. Learn., vol. 3, no. 1, pp. 1-122, Jan. 2011.

[14] Y. Wang, J. Yang, W. Yin, and Y. Zhang, "A new alternating minimization algorithm for total variation image reconstruction," SIAM J. Img. Sci., vol. 1, no. 3, pp. 248-272, Aug. 2008. 